UCRL-ID-146558

\title{
The Advantages of High Order Schemes and How to Confirm These Advantages
}

\author{
L. Jameson
}

November 26, 2001

Lawrence

Livermore

National

Laboratory 
This document was prepared as an account of work sponsored by an agency of the United States Government. Neither the United States Government nor the University of California nor any of their employees, makes any warranty, express or implied, or assumes any legal liability or responsibility for the accuracy, completeness, or usefulness of any information, apparatus, product, or process disclosed, or represents that its use would not infringe privately owned rights. Reference herein to any specific commercial product, process, or service by trade name, trademark, manufacturer, or otherwise, does not necessarily constitute or imply its endorsement, recommendation, or favoring by the United States Government or the University of California. The views and opinions of authors expressed herein do not necessarily state or reflect those of the United States Government or the University of California, and shall not be used for advertising or product endorsement purposes.

This work was performed under the auspices of the U. S. Department of Energy by the University of California, Lawrence Livermore National Laboratory under Contract No. W-7405-Eng-48.

This report has been reproduced directly from the best available copy.

Available electronically at http://www.doe.gov/bridge

Available for a processing fee to U.S. Department of Energy

and its contractors in paper from

U.S. Department of Energy

Office of Scientific and Technical Information

P.O. Box 62

Oak Ridge, TN 37831-0062

Telephone: (865) 576-8401

Facsimile: (865) 576-5728

E-mail: reports@adonis.osti.gov

Available for the sale to the public from

U.S. Department of Commerce

National Technical Information Service

5285 Port Royal Road

Springfield, VA 22161

Telephone: (800) 553-6847

Facsimile: (703) 605-6900

E-mail: orders@ntis.fedworld.gov

Online ordering: http://www.ntis.gov/ordering.htm

OR

Lawrence Livermore National Laboratory

Technical Information Department's Digital Library

http://www.llnl.gov/tid/Library.html 


\title{
The Advantages of High Order Schemes and How to Confirm These Advantages
}

\author{
Leland Jameson \\ Lawrence Livermore National Laboratory
}

\begin{abstract}
This manuscript is meant to give a short summary of the advantages of high order schemes and suitable test problems which can properly illustrate these advantages.
\end{abstract}

\section{The Advantages of High Order Schemes}

The advantages of high order schemes are well-documented in the scientific literature in both the theoretical form and in computational form. It is really the theory that one should focus on since the whole story in its unpolluted form lies there. If one needs computational evidence, then such evidence is copious.

The classic reference is the paper by Kreiss and Oliger [15]. However, here the simplest exposition of the key issues will be given. It is the truncation error of the differentiation operator which holds the.key to the advantages of high order schemes when applied to hyperbolic systems. For non-spectral operators, this truncation error has an upper bound of the form, see [9],

$$
E=c(\Delta x)^{p} k^{p+1}
$$

where $c$ is a constant that will depend on the exact scheme in use, $\Delta x$ denotes the distance between adjacent grid points, $p$ denotes the order of the derivative, and $k$ denotes the Fourier wave number. That is, if one were to differentiate $f(x)=\sin (k x)$ then the truncation error for differentiation is bounded above by the above expression. Now, since,

$$
\Delta x=\frac{L}{N}
$$

where $L$ denotes the length of the interval and where $N$ denotes the number of grid points in this interval, we can let $L=1$, and replace $(\Delta x)^{p}$ with 
$(1 / N)^{p}$. Next, we can now solve equation 1 for $N$. Beginning with

$$
E=(1 / N)^{p} k^{p+1},
$$

where we let $c=1$ for simplicity, we then get,

$$
E^{1 / p}=(1 / N) k^{(p+1) / p}
$$

and

$$
(1 / N)=\frac{E^{1 / p}}{k^{(p+1) / p}}
$$

and finally,

$$
N=\frac{k^{(p+1) / p}}{E^{1 / p}} .
$$

Now, for hyperbolic systems, the floating point operations (flops) scale as,

$$
\text { flops }=\text { const } * O_{\text {time }} * O_{\text {space }} * N^{d+1},
$$

where $d$ is the dimension of the calculation, $O_{\text {time }}$ is the order of the temporal discretization, $O_{\text {space }}$ is the order of the spatial discretization, and where const is a constant that will depend on the scheme used but will not deviate much from .5. By definition $p=O_{\text {space }}$ and for hyperbolic equations $O_{\text {time }}=O_{\text {space }}$ is roughly the best choice. Into this expression for flops we can substitute the above expression for $N$ to get,

$$
\text { flops }=\text { const } * p^{2} * \frac{k^{(d+1)(p+1) / p}}{E^{(d+1) / p}} .
$$

Now, let's see what this expression produces for a few values of the parameters. Let $d=3$ and let's require that the error in the derivative be $E=10^{-4}$. For a first order scheme, $p=1$ we see that this expression becomes,

$$
f \operatorname{lops}(p=1)=10^{8} * k^{8},
$$

and if we let $p=4$ we find that the flops scale as,

$$
f l o p s(p=4)=16 * 10^{4} * k^{5} .
$$

Recall, $k$ is the wavenumber. For flows that contain structure, such as turbulent flows or any calculation where, say, vortices are present, there will 
be significant energy in the high values of $k$. Thus, one can see that the rate of growth of the flops is very different. Further, the constant in front of the expression is also very different. This is really the whole story. With a low order scheme, one quickly reaches the limit of the computer. With the high order scheme, one can obtain far more modes before the limit of the computer is reached.

\section{Designing Problems to Evaluate Schemes}

First and foremost, if one wants conclusive evidence of the differences between schemes, one must design problems that have exact solutions. Without exact solutions one can not have clear evidence of the advantages of high order schemes. It should be noted, that if the flow does not contain structure, then one does not expect to see any advantage of high order schemes. In fact, for problems that are essentially piecewise linear with only a small amount of low order structure in, say, the expansion wave, then one will not see any advantage of high order schemes. This can easily be seen from the derivative. If one differentiates data that essentially lies on a line, then one is wasting flops if the differentiation operator is designed to approximate any polynomial higher than a line.

\subsection{The Role of AMR}

Adaptive Mesh Refinement schemes can reduce work in a computation when the flow variables contain structure that is localized in a small fraction of the computational domain throughout the duration of the computation. If a significant fraction of the domain is filled with structure, then it is not cost effective for adaptive gridding and a uniform grid calculation is preferable. AMR can be a good approach when this criteria is met. Exact estimates of what constitutes a small fraction of the domain for various orders of AMR is discussed in [14].

\subsection{The Value of Convergence Tests}

Numerical calculations are discrete versions of continuous systems. As the number of degrees of freedom increases, this discrete version must approach 
the continuous system it is intended to model. A poorly designed scheme, however, might diverge, or even worse, it might converge to the wrong answer. Thus, a first necessary, though not sufficient condition, is that a numerical calculation converge. Of course, in general we will not have an exact solution, but at least the distance between solutions should decrease as the grid is refined:

$$
|\operatorname{solution}(N)-\operatorname{solution}(2 N)|>|\operatorname{solution}(2 N)-\operatorname{solution}(4 N)| \text {, }
$$

where solution $(N)$ denotes the values of one of the flow variables when there are $N$ grid points in the domain, and $|f|$ denotes a suitable distance measure.

If the calculation converges, then one accepts the calculation at the finest grid point density as the "solution". Without convergence there is no clear concept of what the "solution" is and the calculation does not yield reliable information.

\subsection{Suitable Test Problems}

Here we outline test problems that will yield conclusive information on the value of high order schemes. First of all, for non-linear hyperbolic systems, the derivative is the key numerical operation.

\subsubsection{Linear Convection}

So, in order to isolate this operation we first suggest,

$$
U_{t}=U_{x}
$$

with periodic boundary conditions and with a given single mode as the initial condition:

$$
U(x, 0)=\sin (k x)
$$

This equation is simple and perfect for seeing the value of high order schemes. However, some might insist it is of no value because it is not non-linear. But, if one writes the Euler equations in characteristic variables, and one looks at the local linearization of this system, then along each eigenvector of the linearized system, one obtains linear convection. Hence, this simple equation is an essential and necessary first test problem. If one's scheme does not perform well on this problem, then there is no need to go further. 


\subsubsection{Burgers' Equation}

$\mathrm{Ok}$, then the second suggestion is Burgers' equation,

$$
U_{t}=\left(U^{2}\right)_{x}+\epsilon U_{x x},
$$

with period boundary conditions and an initial condition that is a sine wave shifted up in order to produce a moving shock,

$$
U(x, 0)=1 / 3+2 / 3 * \sin (x) .
$$

This equation has an exact solution and one can even obtain a shock by setting $\epsilon=0$. The two equations alone are sufficient to obtain conclusive evidence of the value of high order schemes. Once one starts testing schemes on calculations that do not have exact solutions, then it becomes very difficult to understand what is numerically produced and what is physical.

\subsubsection{RM and RT calculations}

This is not the regime to test schemes. In other words, the physics is too complicated for one to be able to draw any conclusions about the scheme itself. As stated above, one needs calculations that have exact solutions if one wants to separate the physics from the numerics. RM and RT calculations should be done only after one has a clear understand of the numerical issues.

However, if one does attempt to evaluate schemes using these physical setups one should do the following in order to show the advantage of high order schemes. First, the interface between the materials should have many modes on it and not just one. With a single mode the small structure is limited to a small fraction of the domain and does not clearly show the advantage of high order schemes. Second, the calculation should be run for a long time until the domain is almost full. A principle advantage of high order schemes comes with long-time integration as showed by Kreiss and Oliger cited above. Third, the calculation should be physically symmetric. It will be very difficult to decipher numerical artifacts from physical ones, but loss of symmetry will be one clear sign that a scheme has broken down. Finally, convergence tests must be done. This final point can not be over emphasized. 


\section{Conclusion}

This document is intended to give a guide to the value of high order schemes and how to clearly show this value. As with physical experiments, if the experiment is not clearly thought out then one will never be able to isolate the phenomenon of interest. Certainly this is true of laser experiments and also of numerical experiments.

In the bibliography many references have been given that illucidate high order schemes.

\section{References}

[1] W. Gui and I. Babuska, (1986), "The h-, p- and hp-Versions of the Finite Element Method in One Dimension. Part I: The Error Analysis of the p-Version. Part II: The Error Analysis of the $h$ and hp-Versions. Part III: The Adaptive hp-Version." Numerische Mathematik, Vol. 49, pp. 577-612; 613-657; 659-683.

[2] B. Szabo and I. Babuska, (1991) "Finite Element Analysis", Wiley Interscience.

[3] C. Canuto, M.Y. Hussaini, A. Quarteroni, T.A. Zang, (1988) "Spectral. Methods in Fluid Dynamics", Springer-Verlag.

[4] M. Carpenter and D. Gottlieb, "Spectral Methods on Arbitrary Grids", ICASE Report No. 95-37, NASA CR-198158.

[5] W.S. Don and C.B. Quillen, "Numerical Simulation of Shock-Cylinder Interactions", Journal of Computational Physics, 122, 244-265 (1995).

[6] D. Gottlieb and S.A. Orszag, (1977) "Numerical Analysis of Spectral Methods: Theory and Applications", SIAM-CBMS, Philadelphia.

[7] D. Gottlieb and C.-W. Shu, "On the Gibbs phenomenon III: recovering exponential accuracy in a sub-interval from a spectral partial sum of a piecewise analytic function", SIAM Journal on Numerical Analysis, v33 (1996), pp.280-290. 
[8] D. Gottlieb and C.-W. Shu, "On the Gibbs phenomenon and its resolution", SIAM Review, v30 (1997), pp.644-668.

[9] L. Jameson, "High Order Schemes for Resolving Waves: Number of Points per Wavelength", J. of Scientific Computing, in press.

[10] J. S. Hesthaven and T. Warburton, 2000, "High-Order/Spectral Methods on Unstructured Grids. I. Time-Domain Solution of Maxwells Equations", J. Comput. Phys. - submitted

[11] L. Jameson and T. Miyama, "Wavelet Analysis and Ocean Modeling: A Dynamically Adaptive Numerical Method "WOFD-AHO", Monthly Weather Review, Volume 128, p. 1536-1548.

[12] J. Hesthaven and L. Jameson, "A Wavelet-Optimized Adaptive MultiDomain Method", Journal of Computational Physics 145, 280-296 (1998), Article No. CP986012.

[13] L. Jameson, "A Wavelet-Optimized, Very High Order Adaptive Grid and Order Numerical Method", ICASE Report No. 96-30, and SIAM J. Sci. Comput., Vol. 19, No. 6, pp. 1980-2013, November 1998.

[14] L. Jameson, "AMR vs High Order Schemes", Accepted Journal of Scientific Computing.

[15] H.O. Kreiss and J. Oliger, "Comparison of Accurate Methods for the Integration of Hyperbolic Equations", Tellus XXIV (1972), 3.

[16] H.O. Kreiss, technical report, University of Uppsala, Sweden, 1978.

[17] C.-W. Shu, "Essentially non-oscillatory and weighted essentially nonoscillatory schemes for hyperbolic conservation laws", in "Advanced Numerical Approximation of Nonlinear Hyperbolic Equations", B. Cockburn, C. Johnson, C.-W. Shu and E. Tadmor (Editor: A. Quarteroni), Lecture Notes in Mathematics, volume 1697, Springer, 1998, pp.325-432.

[18] C.-W. Shu, "High order ENO and WENO schemes for computational fluid dynamics", in "High-Order Methods for Computational Physics", T.J. Barth and H. Deconinck, editors, Lecture Notes in Computational Science and Engineering, volume 9, Springer, 1999, pp.439-582. 
[19] W. S. Don, C. Quillen, Numerical simulation of Reactive Flow, Part I : Resolution, Journal of Computational Physics, 122, pp. 244-265, 1995

[20] W. S. Don, D. Gottlieb, Spectral Simulations of Supersonic Reactive Flows, SIAM, Journal of Numerical Analysis, to appear.

[21] R.G. Voigt, D. Gottlieb, Y. Hussaini, (1984) "Spectral Methods for Partial Differential Equations", SIAM. 